\title{
Стимулированное терагерцовое излучение доноров висмута В одноосно-деформированном кремнии при внутрицентровом оптическом возбуждении
}

\author{
(c) Р.Х. Жукавин ${ }^{1}$, С.Г. Павлов ${ }^{2}$, А. Poh/ ${ }^{3}$, Н.В. Абросимов ${ }^{4}$, H. Riemann ${ }^{4}$, B. Redlich ${ }^{5}$, \\ H.-W. Hübers ${ }^{2,3}$, B.H. Шастин ${ }^{1}$ \\ ${ }^{1}$ Институт ффизики микроструктур Российской академии наук, \\ 607680 Нижний Новгород, Россия \\ ${ }^{2}$ Institute of Optical Sensor Systems, German Aerospace Center (DLR), \\ 12489 Berlin, Germany \\ ${ }^{3}$ Department of Physics, Humboldt-Universität zu Berlin, \\ 12489 Berlin, Germany \\ ${ }^{4}$ Leibniz-Institut für Kristallzüchtung (IKZ), \\ 12489 Berlin, Germany \\ ${ }^{5}$ ED Radboud University Nijmegen, Institute for Molecules and Materials, FELIX Laboratory, \\ 6525 Nijmegen, The Netherlands \\ E-mail: zhur@ipmras.ru
}

Поступила в Редакцию 24 апреля 2019 г.

В окончательной редакции 29 апреля 2019 г.

Принята к публикации 29 апреля 2019 г.

\begin{abstract}
Представлены результаты экспериментальных исследований по наблюдению стимулированного излучения в терагерцовом диапазоне частот при оптическом внутрицентровом возбуждении однооснодеформированного кремния, легированного висмутом. Накачка в представленном эксперименте осуществлялась излучением лазера на свободных электронах FELIX. Показано, что одноосная деформация кристалла кремния приводит к значительному изменению спектра стимулированного излучения примеси.
\end{abstract}

Ключевые слова: кремний, висмут, одноосное давление, внутрицентровое оптическое возбуждение, перестройка частоты излучения.

DOI: $10.21883 /$ FTP.2019.09.48140.24

\section{1. Введение}

В последние годы достигнут значительный прогресс в развитии полупроводниковых источников излучения терагерцового диапазона частот [1]. Тем не менее необходимость соответствия требованиям конкретной специализации диктует важность той или иной характеристики источника излучения. Одной из таких характеристик является перестройка линии излучения, которая может быть осуществлена способом, зависящим от физических свойств и характеристик активной среды. В данной работе исследуется влияние одноосной деформации на терагерцовое стимулированное излучение в кремнии, легированном донорами при внутрицентровом возбуждении. В качестве активной примеси был выбран висмут (Bi), имеющий энергию ионизации около 71 мэВ и активный рамановский переход с энергией около 40 мэВ. Стоксов сдвиг для мелких доноров в кремнии определяется разностью энергий между состояниями $1 s\left(A_{1}\right)$ и $1 s(E)$ [2]. Ранее было показано, что оптическое возбуждение доноров V группы в кремнии при низких температурах (ниже $30 \mathrm{~K}$ ) может, в зависимости от кванта возбуждения, приводить к возникновению стимулированного излучения в рамках двух механизмов: инверсии населенности и вынужденного комбинационного рассеяния (ВКР) [3]. В случае фотоионизации Вi рабочими переходами инверсионного механизма являются $2 p_{ \pm} \rightarrow 1 s\left(T_{2}\right), 2 p_{ \pm} \rightarrow 1 s(E)$. Инверсия населенности формируется благодаря специфическому характеру релаксации возбужденных состояний висмута, при котором состояния $1 s\left(T_{2}\right), 1 s(E)$ оказываются относительно незаселенными из-за быстрого „оголения“ состояния $2 p_{0}$ за счет взаимодействия с междолинными оптическими фононами $f$-ТО. В случае внутрицентрового возбуждения наблюдается вынужденное комбинационное рассеяние на переходе $1 s\left(A_{1}\right)-1 s(E)$ в диапазоне квантов накачки, ограниченных снизу энергией перехода $1 s\left(A_{1}\right)-2 p_{0}$, а сверху $-1 s\left(A_{1}\right)-3 p_{0}$ [2]. При этом в случае резонансной накачки могут реализоваться как инверсионный, так и рамановский механизмы и наблюдаться конкуренция этих механизмов [3]. Рамановская активность на переходе $1 s\left(A_{1}\right)-1 s(E)$ обусловлена волновыми функциями $1 s$ состояний, обладающими четностью относительно замены $k_{i}$ на $-k_{i}$, где $i-$ это кристаллографическое направление вдоль оси долины зоны проводимости кремния. С другой стороны, волновые функции триплетного состояния $1 s\left(T_{2}\right)$ нечетные и переход $1 s\left(A_{1}\right)-1 s\left(T_{2}\right)$ в донорах кремния не является рамановски активным. Другим необходимым условием ВКР является существование относительно быстрого канала релаксации $1 s(E)$, что обеспечивается в дан- 


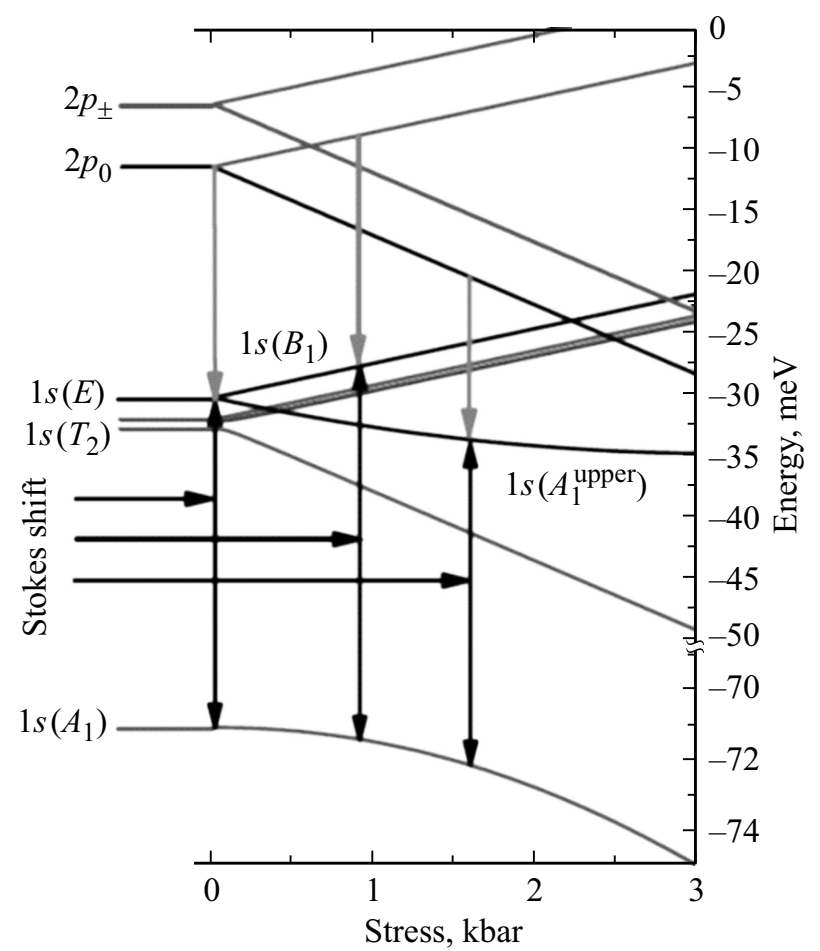

Рис. 1. Расщепление уровней энергии донора висмута в кремнии под действием одноосной деформации вдоль кристаллографического направления [001]. Стрелки вниз показывают квант излучения в случае резонансной накачки состояний донора. Горизонтальные стрелки указывают на стоксов сдвиг, чье изменение вызвано расщеплением и взаимным сдвигом компонент $1 s(E)$ и состояния $1 s\left(A_{1}\right)$. Положение уровней рассчитано в соответствии с работой [5]. Нулевое значение энергии соответствует так называемому центру „масс“ долин и совпадает с дном зоны проводимости в отсутствие давления.

ной системе излучением междолинных фононов LA- $f$. Одноосная деформация кристалла должна привести к расщеплению состояния $1 s(E)$ на две компоненты $1 s\left(B_{1}\right)$ и $1 s\left(A_{1}^{\text {upper }}\right)$, а значит, и к наличию в системе (в определенном диапазоне деформаций для определенного направления) двух активных рамановских переходов (рис. 1). Кроме того, как известно, при деформации изменяются и условия, определяющие инверсию населенности в $\mathrm{Si}: \mathrm{Bi}[4]$.

\section{2. Эксперимент}

Легирование исходного БЗП кремния проводилось методом выращивания с пьедестала [6]. Концентрация висмута в кристалле составляла $\sim N_{D} \sim 3 \cdot 10^{15}$ см. Образец был вырезан в форме прямоугольного параллелепипеда с размерами $7 \times 5 \times 2 \mathrm{Mм}^{3}$ и длинной стороной вдоль направления [001] и „оптическим“качеством полировки граней для формирования высокодобротного резонатора. Образцы $\mathrm{Si}$ : Вi характеризовались путем измерения примесного поглощения при низких температурах ( $5 \mathrm{~K})$ с использованием фурье-спектрометра (Bruker
Vertex 80v) со спектральным разрешением $0.1 \mathrm{~cm}^{-1}$. Эксперимент по наблюдению лазерного эффекта проводился с использованием пользовательской станции лазера на свободных электронах (ЛСЭ) FELIX (Radboud University, Nijmegen, The Netherlands). Импульсы накачки представляли собой макроимпульсы длительностью 6 мкс, следующие с частотой 10 Гц. Каждый макроимпульс состоит из микроимпульсов длительностью $\sim 10$ пс мощностью до 10 МВт, разделенных временны́м интервалом 1 нс. Импульсный характер излучения накачки предполагает, что время жизни фотона в резонаторе по порядку величины не меньше периода повторения микроимпульсов возбуждения. Данное условие достаточно хорошо выполняется для используемых образцов; как показали предыдущие эксперименты, время жизни фотона в резонаторе составляет $\sim 10$ нс [7]. Специальная вставка, содержащая исследуемый образец и позволяющая приложение давления [8], погружалась в транспортный гелиевый сосуд Дьюара. Производилось измерение спектра возбуждения и спектра излучения при различных значениях приложенного давления. Спектр излучения измерялся с помощью фурье-спектрометра со спектральным разрешением до $0.5 \mathrm{~cm}^{-1}$ и сопряженного с фотодетектором $\mathrm{Ge}: \mathrm{Ga}$ (полоса чувствительности с учетом фильтра на основе кристаллического кварца составляла 40-120 мкм). Спектр возбуждения (сигнал германиевого детектора в зависимости от кванта возбуждения) записывался с использованием программного обеспечения пользовательской станции ЛСЭ FELIX.

\section{3. Результаты и обсуждение}

Проведенные эксперименты позволили обнаружить ТГц излучение из $\mathrm{Si}: \mathrm{Bi}$, имеющее пороговую зависимость от интенсивности накачки, что подтверждает его стимулированный характер. Анализ полученных экспериментальных данных позволил выделить в спектре стимулированного излучения висмута в кремнии вклады, имеющие различную зависимость от величины приложенного давления вдоль направления [001]. В отсутствие деформации спектр возбуждения соответствует переходу из основного состояния в состояние $2 p_{ \pm}$, а спектр излучения соответствует переходу $2 p_{ \pm}-1 s(E)$.

Отсутствие более широкого спектра возбуждения в проведенных экспериментах вызвано относительно небольшой мощностью возбуждения и потерями в тракте. Вообще говоря, в такой системе возможно наблюдение рамановской генерации в диапазоне квантов накачки 59-65 мэВ (19-21 мкм). Одноосная деформация приводит к расщеплению линии $2 p_{ \pm}$на две компоненты, что выражается при малых давлениях в появлении аналогичных компонент в спектре возбуждения (рис. 2). При давлении $>200$ бар в спектре возбуждения обнаружены резонансы, соответствующие переходам в компоненты состояния $2 p_{0}$, а в спектре выходного излучения внутрицентровые переходы $2 p_{0}-1 s\left(A_{1}^{\text {upper }}\right)$. 


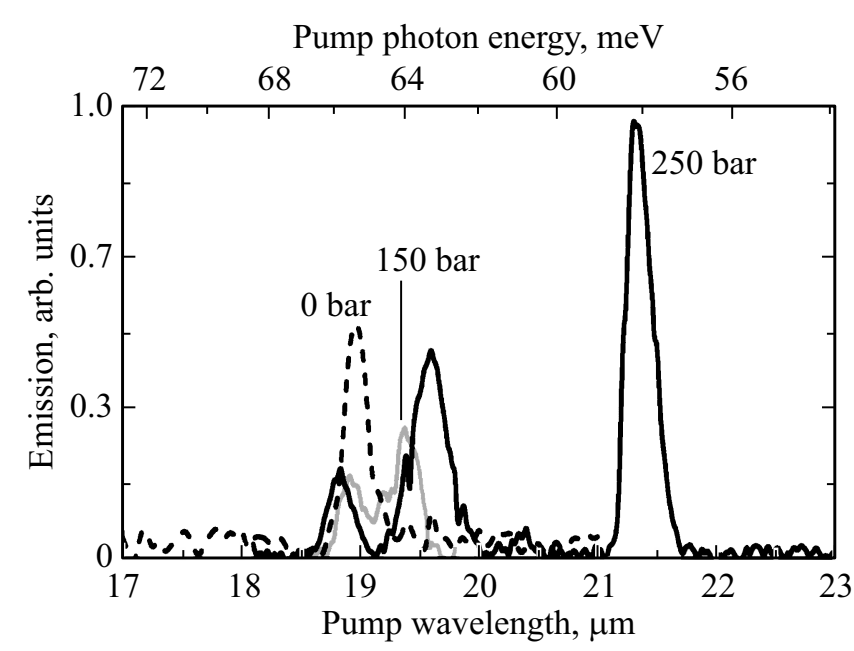

Рис. 2. Зависимость выходной интенсивности $\mathrm{Si}: \mathrm{Bi}$ от длины волны возбуждения для нескольких величин давления вдоль направления [001]. Интенсивность излучения накачки составляла $\sim 1 \mathrm{MB} / \mathrm{cm}^{2}$ в микроимпульсе на входе криогенной вставки. Температура образца $4.2 \mathrm{~K}$.

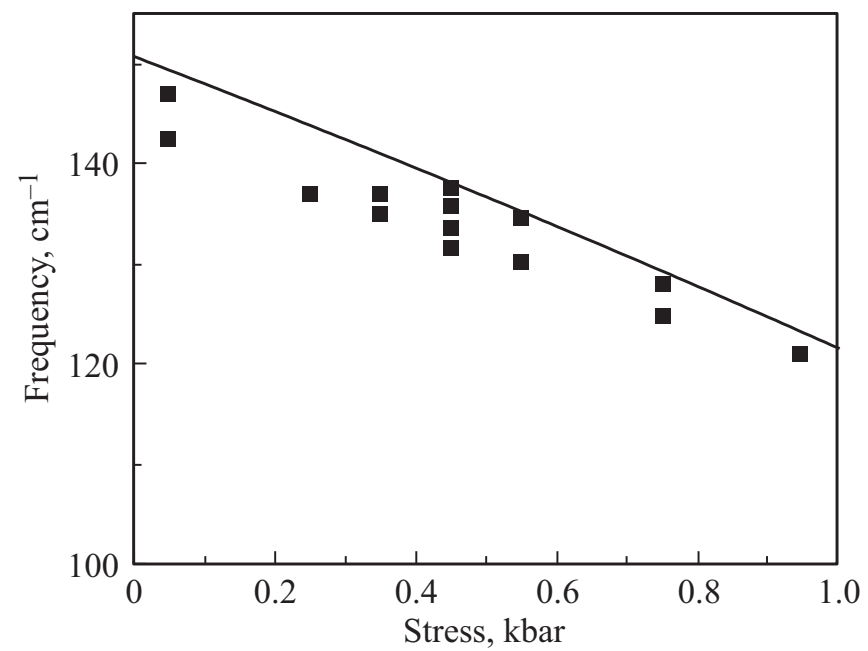

Pис. 3. Зависимость частоты выходного излучения на переходе между нижней компонентой $2 p_{0}$ и $1 s\left(A_{1}^{\text {upper }}\right)$ в зависимости от величины давления вдоль оси кристалла [001] при резонансном возбуждении $2 p_{0}$. Данные эксперимента показаны точками, расчетная зависимость энергии перехода $2 p_{0}-1 s\left(A_{1}^{\text {upper }}\right)-$ сплошной линией.

Обнаружена перестройка частоты излучения на переходе $2 p_{0}-\left(A_{1}^{\text {upper }}\right)$ при увеличении давления, что отражает изменение энергий соответствующего активного перехода. С одной стороны, теоретически величина энергии перехода $2 p_{0}-\left(A_{1}^{\text {upper }}\right)$ в деформированном кремнии может изменяться в довольно широких пределах (рис. 1), однако необходимо также учитывать величину матричного элемента перехода, который должен снижаться при увеличении давления. Последнее вызвано снижением вклада нижних долин в волновую функцию состояния $1 s\left(A_{1}^{\text {upper }}\right)$, однако ввиду значительной вели- чины „химического“ сдвига в висмуте этим обстоятельством можно пренебречь для давлений, используемых в эксперименте. В проведенных экспериментах минимум наблюдаемых частот $\sim 120 \mathrm{~cm}^{-1}$ определяется красной границей фотодетектора на основе $\mathrm{Ge}: \mathrm{Ga}$ и был достигнут для перехода $2 p_{0}-1 s\left(A_{1}^{\text {upper }}\right)$ (рис. 3). Стоит отметить, что в проведенных экспериментах переходы в нижнюю компоненту $1 s\left(T_{2}\right)$ наблюдаются не при всех значениях давления. Данное обстоятельство связано с уширением уровней из-за неоднородности приложенного давления и резонансами с междолинными фононами. Оба эти обстоятельства должны приводить к более сильному подавлению инверсионного механизма генерации. Действительно, эксперименты при фотоионизации донора [7] показали, что для направления одноосной деформации вдоль [001] лазерная генерация отсутствует в диапазоне 1-2кбар [9]. Авторы предполагают, что в случае переходов $2 p_{0}-1 s\left(A_{1}^{\text {upper }}\right)$ ответственным механизмом является вынужденное комбинационное рассеяние света ввиду устойчивости по отношению к неоднородному уширению линий переходов.

\section{4. Заключение}

В данной работе исследовано влияние одноосного давления на терагерцовую лазерную генерацию в кремнии, легированном висмутом, при оптическом внутрицентровом возбуждении. Показано, что одноосное давление приводит к изменению характеристик стимулированного излучения. Получена перестройка линии излучения на переходе $2 p_{0}-1 s\left(A_{1}^{\text {upper }}\right)$ в диапазоне $120-150 \mathrm{~cm}^{-1}$.

\section{Финансирование работы}

Работа поддержана в рамках проекта Российского фонда фундаментальных исследований (19-02-00979) и совместного российско-германского проекта (DFG № 389056032 и 18-502-12077-ННИО). Сотрудники DLR подтверждают, что работа проведена по контракту.

\section{Конфликт интересов}

Авторы заявляют, что у них нет конфликта интересов.

\section{Список литературы}

[1] M. Rösch, G. Scalary, M. Beck, J. Faist. Nature Photonics, 9, 42 (2015).

[2] S.G. Pavlov, R.Kh. Zhukavin, V.N. Shastin, H.-W. Hübers. Phys. Status Solidi B, 250, 9 (2013).

[3] S.G. Pavlov, N. Demann, B. Redlich, A.F.G. van der Meer, N.V. Abrosimov, H. Riemann, R.Kh. Zhukavin, V.N. Shastin, H.-W. Hübers. Phys. Rev. X, 8, 041003 (2018).

[4] В.В. Цыпленков, Р.Х. Жукавин, В.Н. Шастин. ФТП, 48, 1044 (2014).

[5] D.K. Wilson, G. Feher. Phys. Rev., 124, 1068 (1961). 
[6] H. Riemann, N. Abrosimov, N. Nötzel. ECS Transactions, 3, 53 (2006).

[7] R.Kh. Zhukavin, V.N. Shastin, S.G. Pavlov, H.-W. Hübers, J.N. Hovenier, T.O. Klaassen, A.F.G. van der Meer. J. Appl. Phys., 102, 093104 (2007).

[8] R.Kh. Zhukavin, V.V. Tsyplenkov, K.A. Kovalevsky, V.N. Shastin, S.G. Pavlov, U. Böttger, H.-W. Hübers, H. Riemann, N.V. Abrosimov, N. Nötzel. Appl. Phys. Lett., 90, 051101 (2007).

[9] К.А. Ковалевский, Н.В. Абросимов, Р.Х. Жукавин, С.Г. Павлов, Г.-В. Хьюберс, В.В. Цыпленков, В.Н. Шастин. Квант. электрон., 45, (2), 113 (2015). [Quant. Electron., 45, 113 (2015)].

Редактор А.Н. Смирнов

\section{Stimulated terahertz emission of shallow donors in uniaxially stressed silicon under optical intracenter excitation}

R.Kh. Zhukavin', S.G. Pavlov' ${ }^{2}$, A. Pohl' ${ }^{3}$, N.V. Abrosimov ${ }^{4}$, H. Riemann ${ }^{4}$, B. Redlich ${ }^{5}$, H.-W. Hübers ${ }^{2,3}$, V.N. Shastin ${ }^{1}$

${ }^{1}$ Institute for Physics of Microstructures,

Russian Academy of Sciences,

607680 Nizhny Novgorod, Russia

${ }^{3}$ Institute of Optical Sensor Systems,

German Aerospace Center (DLR),

12489 Berlin, Germany

${ }^{3}$ Department of Physics,

Humboldt-Universität zu Berlin,

12489 Berlin, Germany

${ }^{4}$ Leibniz-Institut für Kristallzüchtung (IKZ),

12489 Berlin, Germany

${ }^{5}$ Radboud University Nijmegen,

Institute for Molecules and Materials,

FELIX Laboratory,

6525 ED Nijmegen, The Netherlands

Abstract The results on observation of stimulated terahertz emission from uniaxially stressed silicon doped by bismuth under optical intracenter excitation. The radiation of free electron laser FELIX has been utilized in the presented experiment. It has been shown that uniaxial stress results in significant change of impuritybased stimulated emission spectra. 